\title{
Expulsive Hemorrhage: a Study of Histopathological Details
}

\author{
J. Reimer Wolter \\ Departments of Ophthalmology and Pathology, University of Michigan, Medical Center, Ann Arbor, Michigan 48109, USA
}

\begin{abstract}
Cataract surgery in the eye of a 71 year-old male was complicated by an expulsive hemorrhage. The eye became blind and was removed 21 days later. Histopathological evaluation revealed facts of outstanding interest: rupture of the long posterior ciliary artery on the nasal side was the cause of the massive bleeding and cystoid macular edema with vertical infolding of the central cones was found in the totally detached and distorted retina.

Zusammenfassung. Eine Cataractoperation am Auge eines 71 Jahre alten Mannes wurde durch eine expulsive Blutung kompliziert. Das Auge wurde blind und wurde 21 Tage später entfernt. Histopathologische Untersuchung ergab Befunde von besonderem Interesse: das Platzen der langen hinteren Ciliararterie an der nasalen Seite war der Ursprung der massiven Blutung und zystoides Maculaoedem mit verticaler Einfaltung der zentralen Zapfen wurde in der völlig abgelösten und verzerrten Netzhaut gefunden.
\end{abstract}

The intraocular pressure serves to support the wall of all blood vessels inside the eye. Nature wastes nothing and, thus, all vascular structures in the eye are built with rather thin walls adjusted to the conditions of the usual range of intraocular pressure values. Decompression of an eye has several direct effects on the vascular system: it improves conditions for arterial inflow, it decreases venous outflow, and it eliminates the support that eye pressure gives to the walls of all intraocular vessels. The effect of sudden ocular decompression on ocular arteries can be compared to the situation of deep sea fish quickly brought to the surface: they swell or may even burst.

The central choroid normally has the quickest, the most direct, and most extensive arterial supply of all ocular structures by the way of the posterior ciliary arteries. The posterior choroid is, where fluorescein first appears when it is injected into a vein [1]. The plexus-like structure of the extensive terminal vascular system of the choroid requires relatively large arteries with great velocity of blood flow. The central choroid, therefore, is most severely effected by decompression and the posterior ciliary arteries may become decompensated to a point, that they burst - just like the deep sea fish brought to the surface. A very sudden

Offprint requests to: J. Reimer Wolter. Supported by the Research to Prevent Blindness, Inc., New York and massive hemorrhage deep in the choroid results. This finds room by separating the outer choroidal layer from the sclera at the level of the suprachoroidal space. The resulting massive choroidal detachment is a dramatic natural phenomenon that no eye surgeon ever forgets, when he has seen it. With decompression as a part of most intraocular surgery, the arterial type of choroidal hemorrhage may occur during any operation. It is clinically known as expulsive hemorrhage, because the massive choroidal detachment caused by the arterial hemorrhage suddenly and quickly pushes the ocular contents ahead of it out of the eye. The surgeon will try to make stab incisions in the sclera to release the arterial blood $[2,3]$. With modern microinstrumentation, microscope, and drapes it has become difficult to get to the sclera in time with a sufficiently long knife. The present case report can serve to review the nature and pathology of explusive hemorrhage as well as the location of the arterial rupture in one typical case.

\section{Case Report}

This 71 year-old white male of good general health was operated for a cataract in his right eye on 5-21-1981. He was not hypertensive, but he had used Aspirin for what was called a vertebro-basilar syndrome due to suspected atherosclerosis. The Aspirin was stopped well before the date of surgery. The eye was prepared for surgery with Glycerol by mouth preoperatively and by retrobulbar injection of $2 \%$ Carbocaine with Wydase. Ten minutes of massage were used to obtain hypotony. Implantation of an intraocular lens was planned. Two peripheral iridectomies were done, before the cataractous lens was delivered in its capsule. The iris fell back and a large air bubble separated the cornea from the vitreous face. An estimated 20 minutes after opening of the eye, there occurred sudden expulsion of vitreous - almost immediately followed by retina and blood. This sudden complication took about 20 seconds to develop - and no attempt to drain the suspected choroidal hemorrhage was made. The surgeon describes the event as "the most dramatic thing that I have ever observed." Vitreous and blood were removed. The eye was closed. It continued to bleed, did not have light perception, and became red and painful. It was enucleated on 6-12-81.

The eye was of normal size and had a row of black silk sutures still in place on a recent limbal incision between 9 and 3 o'clock. Opened in a horizontal plane directly below optic nerve and foveal region, the eye revealed blood all 

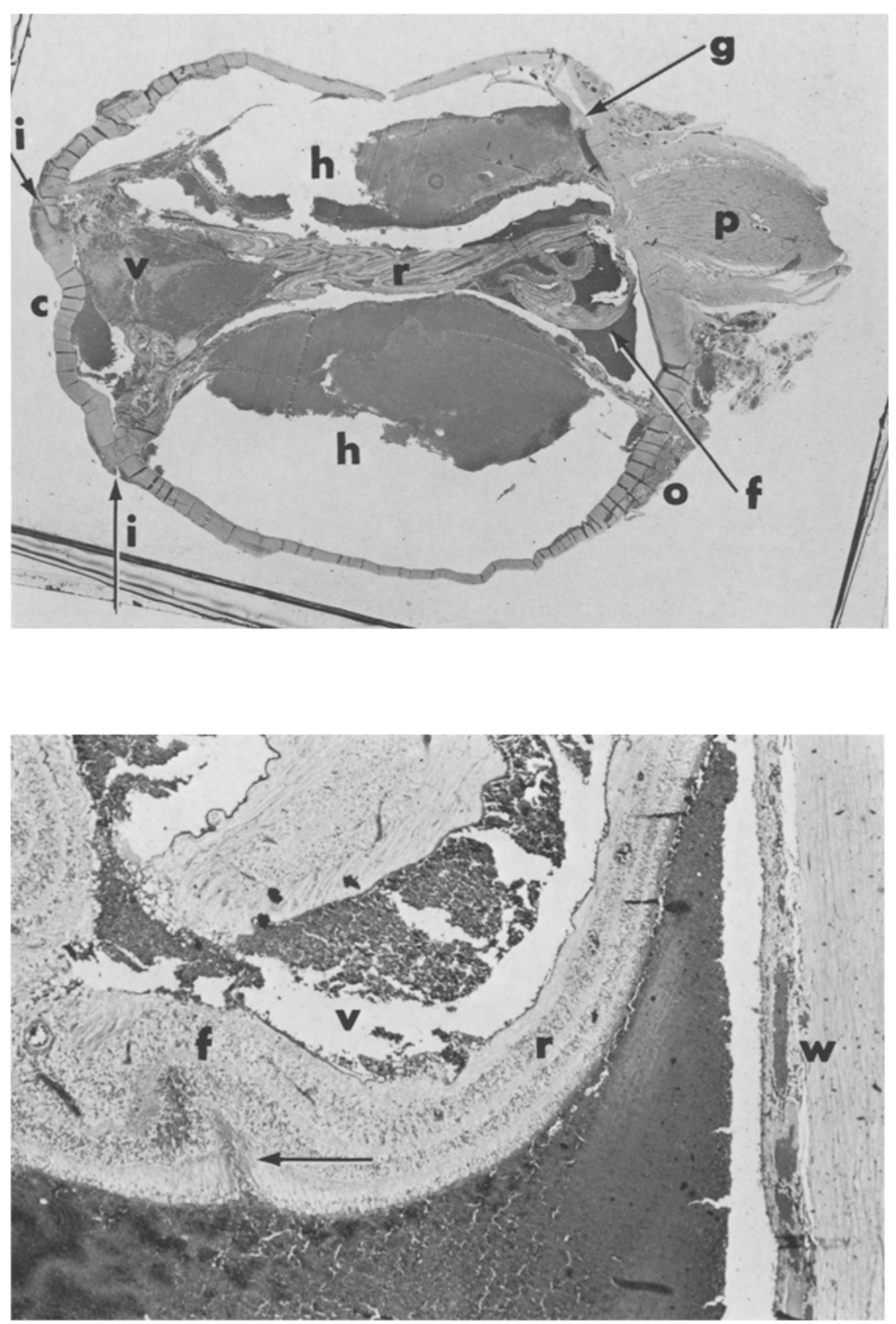

Fig. 1. Section of the whole eye in a horizontal plane. Two arrows point to the recent cataract incision $(i)$. Blood in back of the cornea $(c)$ and vitreous replaced by blood $(v)$ directly adjacent. The retina $(r)$ detached and collapsed. The detached choroid as a continuous layer next to the collapsed retina - separated from its scleral bed by massive hemorrhage $(h)$. The foveal choroid between disk and insertion of the inferior oblique muscle $(o)$ is in place. The swollen foveal retina (arrow $f$ ) separated by blood from this choroid. - Paraffin section, $\mathrm{H}$ and $\mathrm{E}$ stain, photomicrograph $\times 10$

Fig. 2. Parafoveolar retina $(r)$ in horizontal section with sharp vertical fold of outer retinal layers (arrow). Ganglion cell layer $(f)$ well preserved. Blood in vitreous space $(v)$ and in subretinal space. Ocular wall $(w)$ with congested choroid in place and sclera on the right border. - Paraffin section, $\mathrm{H}$ and $\mathrm{E}$ stain, photomicrograph $\times 250$

through its inner parts. Some of the blood was clotted, while other blood was fluid and was lost during the gross examination. The lens was absent. Parts of the totally detached and collapsed retina were seen in the pupillary region. The choroid was detached in the suprachoroidal plane and contained a massive choroidal hemorrhage. On the nasal side, the choroidal hemorrhage extended directly up to the optic nerve. The choroid temporal to the disk - including the foveal zone and the area of the entry of the temporal long posterior ciliary artery into the choroid - was not detached. Massive blood was seen not only in the subchoroidal zone, but it also filled the subretinal space, the vitreous, the space under the detached ciliary body, and the anterior chamber. The subretinal hemorrhage extended under the foveal retina - into that area, where the choroid was not detached.

Microscopic study of routine paraffin sections stained with $H$ and $E$ showed the recent surgical wound with its sutures and in good apposition (Fig. 1). The blood in the anterior chamber contained portions of the distorted iris. The ciliary body was luxated forward on the preserved hinge of the scleral spur and exhibited massive blood under its detached pars plana (Fig. 1). Slight subacute inflammatory reaction associated with much destruction of the pigment epithelium were noted on iris and ciliary body. Pieces of peripheral retina had moved forward into the pupillary zone and some of these were damaged and interrupted. Peripheral choroid was found almost directly in back of these (Fig. 1). Blood filled anterior chamber, remaining vitreous space, retroretinal space, and retrochoroidal space (Fig. 1). Much of the blood of the retrochoroidal space next to the sclera had remained fluid after fixation and had been lost before sectioning.

The choroid and pigment epithelium on the temporal 


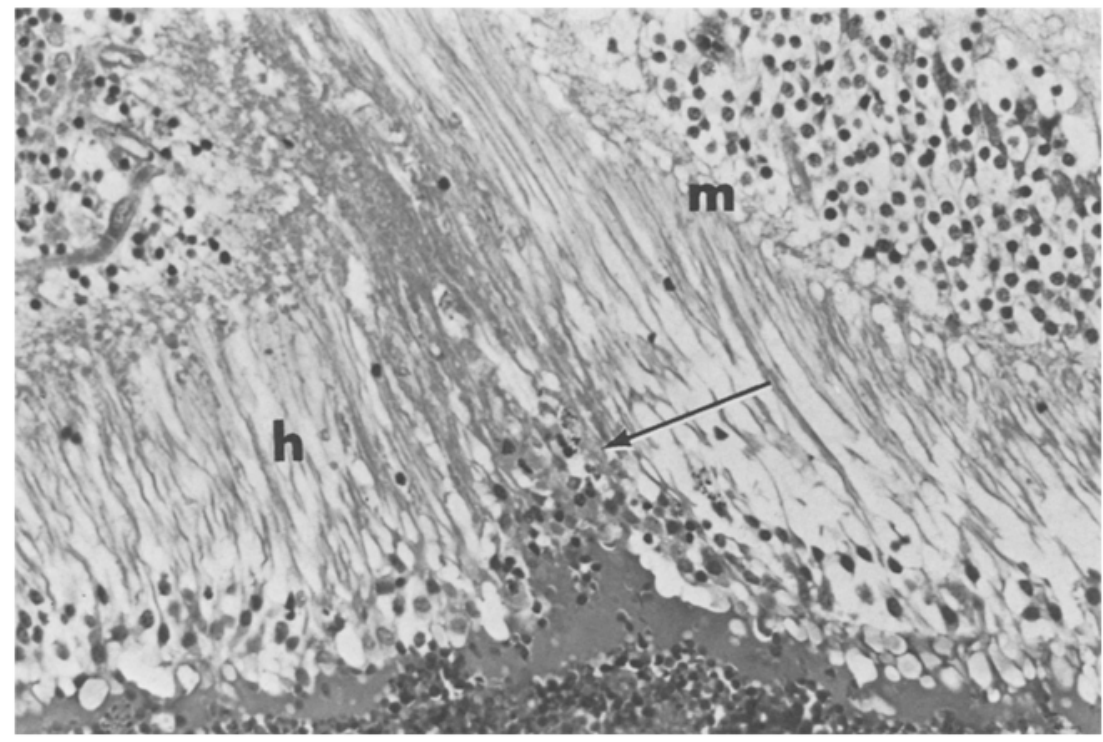

Fig. 3. Microcystoid changes and fragmentation of fibers in foveal Henle's layer $(h)$. Center of the vertical fold of outer retinal layers (arrow) with degeneration of central cones. $m$ : so-called middle limiting membrane.

Paraffin section, $\mathrm{H}$ and $\mathrm{E}$ stain, photomicrograph $\times 750$

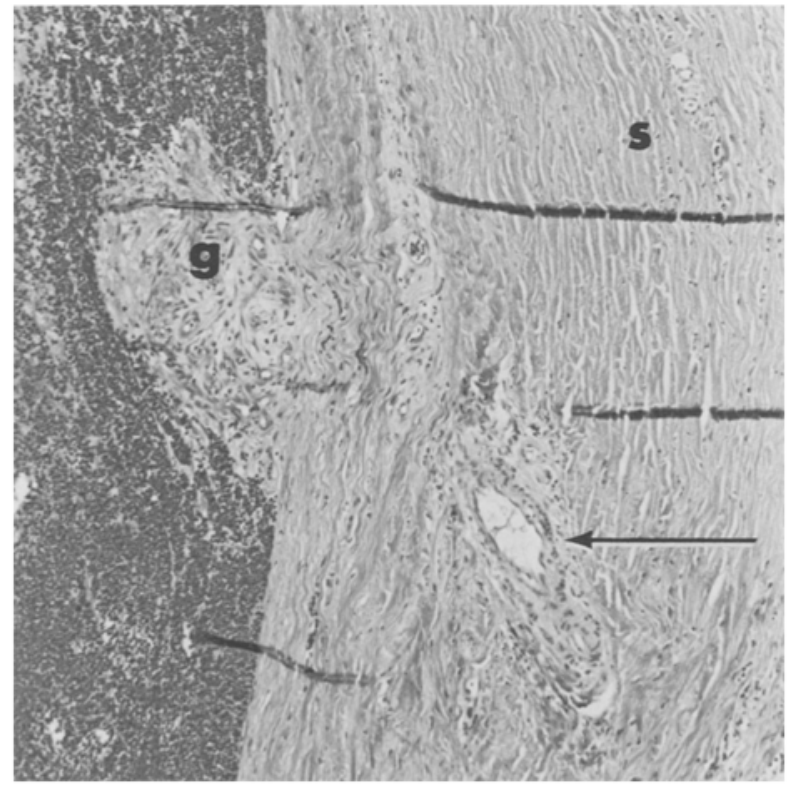

Fig. 4. The granuloma-like scar $(g)$ on the inner scleral exit of the ruptured posterior ciliary artery. The arrow indicates the somewhat shrunken artery in its scleral canal. The sclera $(s)$ on the right side of the view. - Paraffin section, $\mathrm{H}$ and $\mathrm{E}$ stain, photomicrograph $\times 400$

aspect of the disk including the foveal zone were found in place, but a thick layer of blood separated this from the corresponding foveal retina (Fig. 2). The foveal retina was thickened and involved with a deep vertical fold running through the outer layers of its center (Fig. 2). Henle's fiber layer was swollen and exhibited fragmentation of nerve processes and supporting Mueller fiber processes with so-called microcystoid degeneration (Fig. 3). The central cones involved in this vertical fold showed signs of distortion, degeneration, and atrophy (Fig. 3). The inner retinal layers were also distorted due to the detachment, but they were very well preserved in the central area (Fig. 2). The optic nerve head was slightly swollen and the central retinal vessels were open (Fig. 1). The sclera was normal.

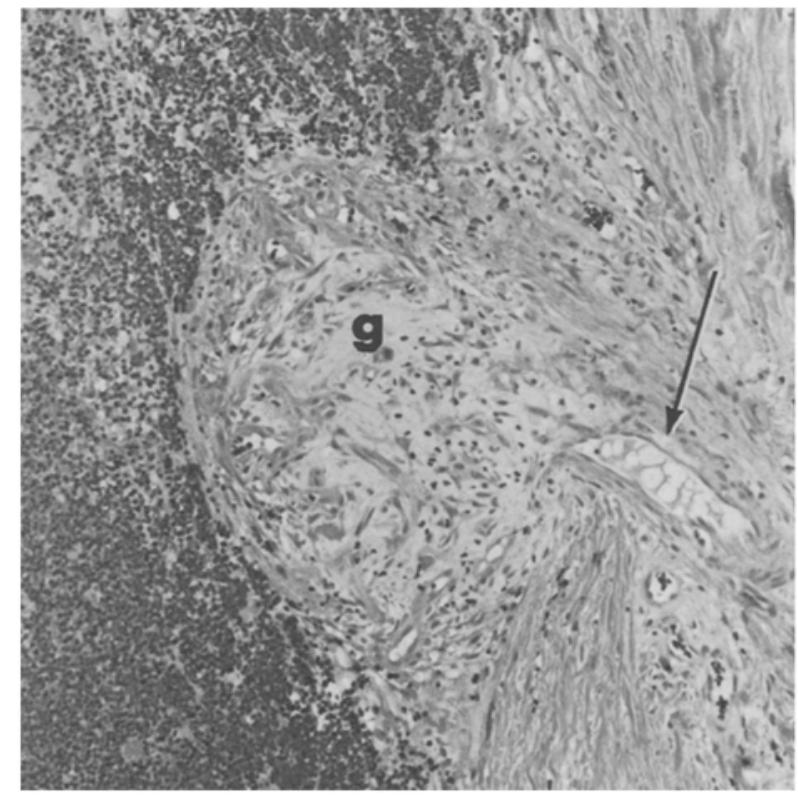

Fig. 5. High power view of the scar $(g)$ on the scleral aspect of the ruptured posterior ciliary artery (arrow) surrounded by dense blood in the suprachoroidal space. - Paraffin section, $\mathrm{H}$ and $\mathrm{E}$ stain, photomicrograph $\times 850$

The long posterior ciliary artery on the nasal side, shrunken somewhat and the blood of its lumen replaced by exudate, was found in the sclera nasal to the optic nerve head (Figs. 1, 4 and 5). This artery was interrupted at its scleral exit and was clearly ripped off the choroid, that was separated from this arterial stump by a thick layer of blood. On the inner scleral surface, the site of this interrupted artery was covered by a reactive scar mainly composed of proliferating fibroblasts and histiocytes (Fig. 5). These findings in addition to the general arrangement of the blood in the suprachoroidal space indicated that rupture of this ciliary artery at its exit from the sclera towards the choroid had caused the expulsive hemorrhage (Fig. 1). 


\section{Discussion}

Expulsive hemorrhage during surgery is rare, fortunately. It is said to occur about once in a thousand cataract operations [4]. It is important to recognize that expulsive hemorrhage can occur during cataract surgery, but it is much more common in chronically inflamed eyes with glaucoma and rupture of a secondary corneal ulcer or after trauma, for example. It has also been reported during surgery for retinal detachment [5] or even spontaneously in an eye with choroidal melanoma [6]. To have a chance, to study an eye with recent expulsive hemorrhage during surgery histologically, is rare. The findings in the present case confirm single earlier observations $[7,8]$. The complication is due to spontaneous rupture of a large choroidal artery. In the present case the long posterior ciliary artery, that ruptured directly at the exit of its scleral canal, did not only burst. It also was ripped off. This interruption may have been secondary - caused by the stretch of the massive arterial hemorrhage that separated the choroid in the plane of the suprachoroidal space. The place, where a ciliary artery exits from its firmly supported scleral canal into the loose structures of the suprachoroidal space, is a very likely location for a rupture, of course. The preformed plane of the suprachoroid, furthermore, allows for the quick expansion of the blood that is typical for expulsive hemorrhages. The arterial rupture did not occur immediately, when the eye was opened. It happened when the operation was almost completed. As explained in the introduction of this paper, this delay is probably due to the slowly additive nature of the components which contribute to the decompensation of the arterial walls: increased inflow, decreased outflow, lack of support of the vessel wall, and extreme congestion. It is generally believed that hypotony induced before surgery by medical and mechanical means gives some protection against the occurrence of expulsive hemorrhage [8]. Review of the events in the present case give no reason to change this view. However, preoperative hypotony may not always be a reliable protection against the slow action of the additive components listed above. The conclusion from all this is, to always aim for the shortest technically possible period of ocular decompression in all intraocular procedures. To operate in a pressure chamber would be the other possibility, of course.

The observation of a well-recognized type of early cystoid macular edema (CME), that exhibits vertical infolding of the outer retinal layers in this case of sudden hemorrhagic retinal detachment in the course of cataract surgery is surprising. This type of CME has been recorded in the attached retina in association with sudden ocular decompression without expulsive hemorrhage [10], (Fig. 3), in a large choroidal melanoma [11] (Fig. 7), in a ciliary body melanoma [12] (Fig. 1), and in an eye with a Choyce type of intraocular lens implant [13], (Fig. 11), for example. The observation of this type of $\mathrm{CME}$ with a vertical fold in the present case stimulates some thought about the question, whether this change can develop in a detached retina separated from the choroid by much blood. The other possibility is that this was already present at 20 minutes of surgical decompression, when the massive hemorrhage occurred and that this was preserved in this state in the detached retina [14]. This question can not as yet be answered. However, the fact that early CME with microcystoid changes in Henle's fiber layer and infolding of the outer retinal layer in a vertical direction can occur in association with expulsive hemorrhage needs to be recorded. Getting to know all the reactive possibilities of the central retina in $\mathrm{CME}$ will slowly result in getting to understand the nature of CME itself.

\section{References}

1. Archer D, Krill AE, Newell FW (1970) Fluorescein studies. Amer J Ophth 69:543-554

2. Verhoeff FH (1915) Scleral puncture of expulsive sub-choroidal hemorrhage following sclerotomy. Ophth Rec 24:55-59

3. Vail D (1938) Posterior sclerotomy as a form of treatment in sub-choroidal expulsive hemorrhage. Amer J Ophth $21: 256-260$

4. Holland $\mathrm{G}$ von (1966) Zur Klinik der expulsiven Blutung nach Kataraktoperation. Klin Mbl Augenheilk 149:859-864

5. Wolter JR (1961) Expulsive hemorrhage during retinal detachment surgery: A case with survival of the eye after Verhoeff sclerotomy. Amer J Ophth 51:264-266

6. Perry HD, Hsieh RC, Evans RM (1977) Malignant melanoma of the choroid associated with spontaneous expulsive choroidal hemorrhage. Amer J Ophth 84:205-208

7. Manschot WA (1955) The pathology of expulsive hemorrhage. Amer J Ophth 40:15-24

8. Freedman J (1981) Posterior sclerotomy: A surgical approach for lowering intraocular pressure. Ophth Surg 12:347-348

9. Winslow RL, Stevenson W III, Yanoff M (1974) Spontaneous expulsive hemorrhage. AMA Arch Ophth 92:33-76

10. Wolter JR (1981) Vertical folds of central retina and choroid in sudden ocular decompression. Ophth Surg 12:190-194

11. Wolter JR (1982) Three basic types of foveal involvement in choroidal melanomas. Graefe's Arch Ophth 218:237-243

12. Wolter JR (1982) Foveal pathology in ciliary body melanoma. Ophth Surg 13:309-311

13. Wolter JR, Croasdale RE, Bahn CF (1980) Reactions to an anterior chamber lens, two years after implantation. Ophth Surg 11:794-800

14. Wolter JR (1981) The histopathology of cystoid macular edema. Graefe's Arch Ophth 216:85-101

Received July 5, 1982 